\title{
Adolescent Sexual and Reproductive Health Counselling in Relation to Female Genital Cutting: Swedish Professionals' Approach to Menstrual Pain as an Empirical Example
}

\section{Camilla Palm $^{1,2}$ D $\cdot$ Sara Johnsdotter ${ }^{1}$. Eva Elmerstig ${ }^{1} \cdot$ Charlotta Holmström $^{1}$. Birgitta Essén ${ }^{2}$}

Accepted: 3 July 2021 / Published online: 14 July 2021

(c) The Author(s) 2021

\begin{abstract}
In Sweden, as well as in an international context, professionals are urged to acquire knowledge about possible health effects of female genital cutting (FGC) in order to tackle prevention and care in relation to the practice. While professionals are guided by policies and interventions focusing on medical effects of FGC, some scholars have cautioned that many popular beliefs about health risks rest on inconclusive evidence. The way professionals understand and respond to health information about FGC has in this context largely been left unexamined. This article aims to provide a qualitative exploration of how professionals in Sweden approach adolescent sexual and reproductive healthcare encounters in relation to acquired knowledge about FGC, using menstrual pain as an empirical example. The analysis shows that there was a tendency in counselling to differentiate young migrant women's menstrual complaints from ordinary menstrual pain, with professionals understanding pain complaints either in terms of FGC or as culturally influenced. The study shows how professionals navigated their various sources of knowledge where FGC awareness worked as a lens through which young women's health complaints were understood. Biomedical knowledge and culture-specific expectations and assumptions regarding menstrual pain also informed counselling. Finally, the article discusses how FGC awareness about health risks was used constructively as a tool to establish rapport and take a history on both menstrual pain and FGC. The analysis also recognises potential pitfalls of the approaches used, if not based in well-informed policies and interventions in the first place.
\end{abstract}

Keywords Female genital cutting - Sexual and reproductive health counselling . Menstrual pain $\cdot$ Migration $\cdot$ Youth $\cdot$ Sweden

Camilla Palm

camilla.palm@mau.se

Extended author information available on the last page of the article 


\section{Introduction}

Female genital cutting (FGC), also referred to as female genital mutilation (FGM), ${ }^{1}$ involves a range of genital alteration procedures performed on girls for non-medical reasons. Procedures are categorised by the World Health Organization (WHO) into four main types: excision of the glans clitoris and/or clitoral hood (type I), excision of the labia (type II), narrowing of the vaginal opening after cutting and suturing the labia minora, sometimes combined with clitoridectomy (infibulation, type III), and type IV (unclassified) including pricking, nicking, scraping and cauterisation (WHO, 2018). It occurs primarily in some groups in Sub-Saharan Africa and in parts of Middle East and Southeast Asia, and circumstances under which the operation is performed vary widely. Also motives vary with locality and may include social acceptance, preparation for adulthood and marriage, marking of ethnic/religious/gender identity, preservation of cleanliness and modesty, and aesthetical preference (Ahmadu, 2000; Jirovsky, 2014; Leonard, 2000; Powel et al. 2020).

In policy and guidelines for professionals, in Sweden and globally, addressing possible medical sequelae of FGC has continuously been emphasised as a means to prevent the practices from being perpetuated and to optimise care and support for girls and women with FGC (e.g. Ministry of Health \& Social Affairs, 2018; WHO, 2008; WHO, 2016; Östergötland County Administrative Board, 2015).

Meanwhile, several scholars have noted that research on health risks possibly related to FGC does not provide a clear picture (e.g. Obermeyer, 2005; PPAN 2012). In many cases, evidence of sexual and reproductive health outcomes is inconsistent (see reviews by Berg \& Denison, 2012; Johnsdotter, 2018; Obermeyer, 2005). Whereas many studies highlight increased health risks and, for example, have found a higher likelihood of reportings of genital pain (Lurie et al., 2020) or less sexual satisfaction (Berg \& Denison, 2012), other studies on sexuality document frequent reports of orgasm, desire and satisfaction (Abdulcadir et al., 2016; Catania et al., 2007; Obermeyer, 2005) and no statistically significant differences on genital pain between women with or without FGC (Alsibiani \& Rouzi, 2010). This great variation underscores the challenges in conducting research on sexual health outcomes related to FGC. These challenges are both methodological and conceptual. As highlighted by Obermeyer (2005) in her review, many of the existent studies suffer from methodological shortcomings such as small research samples and lack of appropriate control groups. Many descriptions of presumed health effects also fail to discriminate between different forms of procedures or how they are performed e.g. in a medicalised setting, by a medically trained person at a hospital or at home, or under less sterile conditions (Obermeyer, 2005; Earp \& Johnsdotter, 2021; PPAN 2012). ${ }^{2}$

\footnotetext{
1 What to call the non-western forms of genital alteration procedures is debated. While WHO uses 'mutilation', it is conceded that such a term may be stigmatising, not least in a clinical context (WHO 2018). In this article, the term 'FGC' will be used to refer to all practices included in the WHO typology. Other descriptive terms will also be used.

2 Some also contend that representations of health risks are culturally biased, as they focus solely on non-western forms of FGC although there are forms of FGCs that in a strict clinical and anatomical sense are comparable to medically unnecessary surgeries on children generally accepted in the West; i.e.,
} 
Another challenge is the lack of appropriate theoretical concepts to properly capture subjective feelings of pain or pleasure, when such experiences are highly embedded in cultural meanings, values and ideals (see e.g. Dopico, 2007 or Jirovsky, 2014 for empirical examples). Consequently, these factors limit the generalisability of medical claims commonly presented in policy and public-health research on FGC, and make it difficult to establish the actual medical effects of FGC on sexual and reproductive health (Shell-Duncan, 2008).

Against this background, a central question is how professionals navigate and manage sexual and reproductive health in care-encounters with girls and women with FGC, when they are positioned between FGC policy and interventions on the one hand, and uncertain research evidence on the other. Previous research among professionals in western countries has focused primarily on care providers' knowledge, attitudes and practices (KAP). Reviews show professionals lack confidence and experience in caring for women with FGC, reporting a variation in FGC training among care providers (see reviews by National Board of Health \& Welfare, 2020; Reig-Alcaraz et al., 2016). To date, the way professionals understand and respond to knowledge gained from FGC training, campaigning and information, including awareness of potential health risks, has not been studied. This article aims to explore how professionals in schools, healthcare and social services approach sexual and reproductive healthcare encounters in relation to acquired knowledge about FGC. Using menstrual pain as an empirical example, the article demonstrates how professionals navigate their different sources of knowledge in counselling with young women potentially affected by FGC. Ultimately, the study also serves as an example of the impact of FGC policy and interventions on work practices, a question which merits further critical examination.

\section{Painful Menstruation in Relation to Female Genital Cutting: Where Does the Evidence Stand?}

Pain associated with menstruation, known as dysmenorrhoea, is commonly separated into pain occurring with menstruation in the absence of pelvic pathology (primary dysmenorrhoea), or pain occurring with menstruation caused by other underlying pelvic conditions such as endometriosis or pelvic inflammatory disease (secondary dysmenorrhoea) (Iacovides et al., 2015). In primary dysmenorrhoea, studies have found pain to derive from several factors, particularly: excessive uterine contractions under the influence of prostaglandins (Fajrin et al., 2020), contractions of uterine blood vessels with decrease in uterine blood flow (ischaemia) and reduced oxygen to the uterus (hypoxia), and the stimulation of pain neurones (Iacovides et al., 2015). In severe dysmenorrhoea, an association with high levels of prostaglandins has been reported, and prostaglandins are implicated also in secondary

Footnote 2 (continued)

circumcision of boys, genital cosmetic surgeries on minors, and 'normalising' intersex surgeries on children (Brussels Collaboration on Bodily Integrity 2019). 
dysmenorrhoea (Harel, 2012; Iacovides et al., 2015). Medical treatments recommended in research and guidelines for professionals include analgesics or hormonal contraceptives to reduce prostaglandins, while treatment of secondary dysmenorrhoea can include treatment with medication or other treatment of the underlying condition (ACOG, 2021; Harel, 2012).

In policy papers and research on FGC citing potential health risks, it is often claimed that FGC causes menstrual pain (e.g. Alsibiani \& Rouzi, 2010; Nour, 2008; WHO, 2016, 2020, 2021), though few studies have examined this alleged association or its causes in detail. The rationale for the stated outcome is seldom clarified, although two hypotheses dominate in the literature: that a narrow vaginal opening (FGC type III) may lead to obstruction of menstrual flow and haematocolpos and haematometra (i.e. accumulation of blood within the vagina and uterus) or that FGC may give rise to infections which ascend to the internal genitalia resulting in inflammatory disease and scarring and subsequent painful menstruation (Abdulcadir et al., 2011; Elnashar \& Abdelhady, 2007; Iavazzo et al., 2013; Nour, 2008; WHO, 2018). The hypothesised causality has not yet been verified through empirical studies. Among the few studies that have examined pelvic infections in relation to FGC, none has found an association between the two (see reviews by Iavazzo et al., 2013; Obermeyer, 2005). The hypothesis about an accumulation of blood seems to be deduced from the condition 'imperforate hymen' (a total closure of the vagina) where blood is accumulated in the internal genitals. Whether this condition can be extrapolated to individuals with FGC type III, infibulation (where there is still a vaginal opening), has not been confirmed through empirical studies or clinical observation, i.e. ultrasound or clinical findings of retained menstrual fluid at defibulation surgeries.

On closer examination, a review of the literature shows that sources supporting specific claims about menstrual pain do not always provide evidence in favour of the claim, and no apparent original research forming the basis of the claim can be found. One example illustrating this tendency is the systematic review by Iavazzo and colleagues (2013), cited in the WHO guidelines for professionals (WHO, 2016). With referral to four reviews, the authors claim that FGC causes painful menstruation 'due to obstructed blood flow' (Iavazzo et al., 2013, 1146). However, an assessment of the sources cited shows that one review does not address menstrual pain at all (i.e. Momoh, 2004), or that sources cited in the reviews, respectively, either contradict the claim such as the Gambian study (Morison et al., 2001 in Obermeyer, 2005) that found higher frequencies of menstrual pain among women not exposed to FGC compared to women with FGC, or refer to empirical studies or advocacy literature that has not studied menstrual pain (such as many of the sources cited in Nour, 2004, 2008).

Where does this leave the evidence on menstrual pain and FGC? Empirical studies on health outcomes after FGC show that the evidence on the potential link between various forms of FGC and menstrual pain is inconclusive. Setting aside the limitations in the academic publications discussed above, existing research shows disparate results. For example, some studies conducted among groups where FGC type I-II are common have found statistically significant higher frequencies of painful menstruation among women with FGC compared to the control group of women without FGC (El-Defrawi et al., 2001; Elnashar \& Abdelhady, 2007; Kaplan et al., 
2013; Mahmoud, 2016; Shiferaw et al., 2014), whereas other studies found no such difference between the groups (Andro et al., 2014; Arafa et al., 2018; Morison et al., 2001). Likewise, a recent systematic review and meta-analysis of painful gynaecological complications summarises that 'there was insufficient evidence to conclude that $\mathrm{FGM} / \mathrm{C}^{3}$ is associated with dysmenorrhea' (Lurie et al., 2020, 3). In studies among women with FGC type III (Catania et al., 2007; Chalmers \& Hashi, 2000; Momoh et al., 2001), documented frequencies of menstrual pain range from $44 \%$ (Catania et al., 2007) to 74\% (Chalmers \& Hashi, 2000). Severity of pain is seldom reported. The figures can be compared with occurrences reported in the general population, varying from 33 to $93 \%$ across age groups (see global reviews by De Sanctis et al., 2016; Latthe et al., 2006) and 89\% in a sample of young Swedish women (Söderman et al., 2019). About $15 \%$ of adolescents report severe dysmenorrhoea leading to absenteeism from work/school and social activities (De Sanctis et al., 2015; Söderman et al., 2019). Differences in estimates may be due to how pain is defined and different ways of measuring pain (De Sanctis et al., 2016). Differences may also be attributed to contextual factors such as the varying cultural values or meanings ascribed to menstruation, suffering and pain by respondents in different settings (see e.g. Bobel, et al., 2020), although such factors are seldom discussed. Nonetheless, the overall impression is that dysmenorrhoea is a very common condition in populations with or without any type of FGC.

Taken together, the previous research on FGC does not provide support for the assumption that FGC is evidently related to menstrual pain, let alone causing menstrual pain. Yet, qualitative studies with infibulated women (Abdi, 2012; Thierfelder et al., 2005; Villani et al., 2016) and with providers caring for women with FGC type I-III (Carven et al., 2016; Thierfelder et al., 2005) have documented menstrual pain as a common problem reported in relation to FGC. Whether this is a result of dysmenorrhoea being a very common condition among all menstruating persons, or if there is an actual causal link to FGC (which would possibly require a different treatment of menstrual pain) has not been established and calls for further studies.

\section{Health Claims in FGC initiatives in Sweden}

Since the 1980s, families have migrated to Sweden from the Horn of Africa, where the practice of FGC types II and III have been almost universal (UNICEF, 2013). A number of initiatives have since been mediated through Swedish government programmes aimed at expanding knowledge of FGC, including guidelines, handbooks, a national helpline, awareness-raising activities targeting affected communities, professionals and the general public, and national support for local initiatives in the work against FGC (Östergötland County Administrative Board, 2016). For example, the Swedish government has commissioned the agencies responsible for the national work on FGC to disseminate guidelines and knowledge produced within the ongoing FGC programme (Östergötland County Administrative Board, 2016).

${ }_{3} \mathrm{FGM} / \mathrm{C}$ is an abbreviation for female genital mutilation/cutting, our note. 
In 2015 alone, over 5,000 professionals in welfare and civil society organisations were reached through this specific FGC education, and there has been an ongoing interest in these activities from welfare organisations ever since (Ministry of Health \& Social Affairs, 2018; Östergötland County Administrative Board, 2016). The education covers recommended ways to broach the topic in clinical encounters, ways to work preventively, as well as background information on FGC including descriptions of different types of FGC, where cutting is prevalent, various motives, enumeration of consequences, and information on legislation (Östergötland County Administrative Board, 2015, 2016). Reference material on FGC, produced within the national program, is also available online in the form of presentations, information material, fact sheets and e-learning on official platforms.

A core feature of awareness-raising and education for professionals has been to address potential harmful effects of FGC. The intention, as stated in policy and guidelines, is to enable professionals to identify and treat complications and to discourage the practices by providing information about the potential harm of FGC (Ministry of Health \& Social Affairs, 2018; National Board of Health \& Welfare, 2016; Östergötland County Administrative Board, 2015). In the Swedish context, menstrual pain and school absenteeism due to severe menstrual pain, have been highlighted in reference materials and education materials, as well as by government appointed experts in news media ${ }^{4}$ as one of many symptoms for professionals to be watchful for (Essén \& Johnsdotter, 2017). Likewise, Swedish nursing and medical school literature suggests that FGC 'should be expected' if a young woman from an FGC-practising country presents with menstrual pain (Almroth, 2008, 100). In these descriptions, the rationale is not always explained, although haematocolpos, genital scarring or pelvic infections are sometimes mentioned (National Board of Health \& Welfare, 2016; Östergötland County Administrative Board, 2015). However, these hypotheses have not been tested empirically. Awareness-raising initiatives have thus contributed to diffusing quite a uniform but empirically unsupported representation of menstrual pain in girls and women with FGC.

Sweden has a long history of FGC-related work. Hence, professionals in Sweden have long been subjected to extensive information and awareness-raising activities of FGC-information which highlights sometimes uncertain medical consequences, such as menstrual pain. This context makes an interesting empirical example for studying professional practices in relation to awareness-raising interventions and acquired knowledge about FGC.

\footnotetext{
${ }^{4}$ Government-appointed experts working in the programmes have been cited in various news media mirroring features of their guidelines on FGC: 'They [girls] particularly call [the helpline] during the winter when their pain is at its worst. When it's cold, the menses clot and are difficult to expel if the girl is sewn closed' [Expert cited in large Swedish newspapers, SVT; TT 9 June 2016]. Needless to say, a process such as this - that menstrual fluid inside the body would be affected by outdoor temperature - is not a physiological possibility.
} 
Table 1 Composition of participants according to profession and workplace (n)

\begin{tabular}{lllllc}
\hline & School & Social services & Healthcare & County level & Total \\
\hline $\begin{array}{l}\text { Medical providers (nurse/ } \\
\text { midwife/physician) }\end{array}$ & 12 & 1 & 3 & & 16 \\
$\begin{array}{l}\text { Social worker } \\
\text { Health educator* }\end{array}$ & 1 & 5 & & 1 & 6 \\
Total & 1 & & & 2 \\
\hline
\end{tabular}

*Health educator refers here to people who coordinate intervention programmes [utbildningssamordnare], including FGC education targeting migrant groups and professionals at either local or county level

\section{Methods}

\section{Participants and Recruitment}

The present study emerged from a broader qualitative project ${ }^{5}$ on sexual health counselling and sexual health among women with FGC living in Sweden, with a particular focus on young women and professionals providing care and support for this group.

Twenty-four professionals were included in the study. They worked as nurses, physicians, midwives, health educators [utbildningssamordnare] and social workers in different settings such as schools, primary care, youth clinics, residential homes for children or social services across the country. The majority were healthcare providers in clinical practice. A description of participants according to profession and workplace is provided in Table 1. Professionals worked with young people aged 13-23 years, a few only with minors whereas most encountered both young women who are minors and women over 18, and thus adult by definition. 'Young women' will hereafter be used to refer to the young people the professionals worked with, even though this does not exclude girls under 18. All participants included in the study were women. None had a background in a region where FGC is customary.

While consultation on sexual and reproductive health matters for adolescents in Sweden is mainly offered by youth clinics or within school healthcare, the national strategy on FGC is not limited to medically trained professionals, but includes a broad spectrum of personnel such as school staff and medical and social care providers. In this setting, a number of professions can be expected to be involved in targeted interventions on sexual and reproductive health for girls and women with FGC. Eligible to participate in the study were professionals who gave their informed consent and who had experience providing sexual and reproductive health counselling for young women with FGC.

\footnotetext{
5 This study is part of a Ph.D. project titled Sexual health among Somali women with female genital cutting living in Sweden.
} 
To recruit participants, the project was advertised in various settings including two interdisciplinary networks addressing sexual and reproductive health and rights issues (SRHR), and through one of Malmö University's recurring open seminars on SRHR for interdisciplinary groups of professionals. Emails were also sent to professionals in residential areas or institutions with significant migrant youth populations: i.e. youth clinics, preparatory school programmes and health-related reception services for newly arrived young people. ${ }^{6}$ Participants were also recruited through subsequent snowballing. With only two exceptions, all participants had taken part in FGC education activities and reference materials on FGC. This probably reflects the recruitment strategy, which particularly targeted professionals who felt that they had experience to contribute. It probably also reflects the Swedish context, where professionals working with migrants are likely to have been offered FGC education.

\section{Procedure and Analysis}

This article draws on an analysis of sixteen individual qualitative interviews and three focus group discussions (FGDs) conducted by the first author from 2016 to 2020. Interviews were semi-structured with open-ended questions, and focused on sexual and reproductive health encounters with young people in relation to FGC. Individual interviews ranged from 60 to $80 \mathrm{~min}$. FGDs comprised three participants each and lasted from 90 to $120 \mathrm{~min}$. One professional who participated in an FGD was also interviewed individually. Interviews took place at the professionals' workplaces, or by telephone if preferred by the interviewee. One interview was conducted at a café. All were audio-recorded, except one individual interview during which notes were taken. All recorded interviews were transcribed verbatim.

The analysis followed what Braun and Clarke (2006) call an inductive thematic analysis approach. The analysis began with open coding where salient themes and recurring phenomena were identified through repeated reading of the transcripts. Through this process, approaches to menstrual pain was identified as a prominent theme in the material, which could add empirical insights to existing knowledge in the research field. Instances in the material that were deemed of relevance to this theme were tentatively coded, and subsequent themes and sub-themes were developed. Additional interviews were conducted to explore aspects of interest in more depth. During the analytical process, themes and sub-themes were then reviewed and refined during the process of writing.

\footnotetext{
${ }^{6}$ It is important to note that many young women from groups where FGC is prevalent have undergone FGC prior migration to Sweden, and research supports that there is a general abandonment of FGC after migration (Johnsdotter 2019). Thus, professionals in Sweden who have encountered FGC in their work have likely worked with immigrated girls and young women.
} 


\section{Results}

Three themes were identified in the thematic analysis in terms of professionals' approaches and strategies in relation to menstrual pain in young women with FGC: (1) 'Female genital cutting as a presumed cause of menstrual pain: medical explanatory frameworks'; (2) 'A constructivist approach to menstrual pain' (3); 'Menstrual pain as a working tool in clinical practice'.

\section{Female Genital Cutting as a Presumed Cause of Menstrual Pain: Medical Explanatory Frameworks}

Most professionals presented menstrual pain as a common health consequence of FGC. Participants without a medical background referred to information obtained from FGC education, while medical professionals additionally supported their arguments with reference to medical explanatory frameworks in terms of their clinical knowledge of menstrual characteristics. Medical professionals described typical menstrual pain as originating from uterus activity, either as cramps from uterine contraction when the mucosal tissue discharges, or as lack of oxygen to the uterus. Menstrual pain in relation to FGC was discussed in terms of disturbances from these physiological processes.

A main idea was that FGC causes menstrual pain due to obstructed outflow. Interviewees expressed this as menstruation 'accumulating' in the body, saying it 'doesn't come out', or 'is left in the abdomen'. A health educator commented:

It's the classic thing, you know: stomach aches and blood accumulating ... but then the question is what is left there from before? After all, not everything will come out. ... I guess you've seen, you've read about this, haven't you? How they can get large accumulations of blood in the abdomen, that it basically can be the size of a child's head.

Some explicitly referred to FGC information from awareness-raising activities as sources of their understanding. One social worker, for example, commented that she particularly valued information about signs of FGC to look out for, including 'blood accumulating, and that they get clots [of blood] in the abdomen and those kinds of complications that you might not have thought of in the first place'. These descriptions of 'clots of blood', or 'blood accumulating' in the body, reflect how menstrual pain has been framed in public-health information on FGC in Sweden (see also WHO, 2018). In expressing these ideas, professionals implicitly equated 'FGC' with a narrow vaginal opening. Many, however, noted that it turned out young women seldom had infibulation, but often reported other variations of FGC equal to type I-II or sometimes 'pricking'. This is in line with research in Somalia, showing trends towards lesser operations among the Somali group (Powell et al., 2020) which is the largest migrant group in Sweden from a community where FGC is customary. The Somali group was also the group repeatedly referred to in the professionals' accounts. 
Although participants asserted that obstructed menstrual flow could cause pain, their reasoning showed that it was not clear how this association was to be understood. When asked to clarify, medical professionals drew on their clinical knowledge of dysmenorrhoea, suggesting a number of different possibilities. Some attributed pain to disruption in and prolonging of uterus activity associated with primary dysmenorrhoea. One example of this was expressed by a midwife working as a school nurse:

The uterus has to work hard to get rid of the blood, cramping, contracting more in order to discharge it ... and it becomes like a clot that won't come out ... it will come out eventually, but this of course makes it much more painful.

Likewise, another midwife and school nurse described menstrual pain as linked to how fast the mucosal tissue could discharge. Being partially sewn closed could cause clots, which in turn prolonged the painful process, she reasoned. Others described the pain as a result of blood accumulating in the uterus, cervix and/or vagina, causing 'pressure and aching' in the genital area. Yet some gave no further explanation, or said they were uncertain how to explain the association. In these manners, medical explanatory frameworks about 'normal' or 'disrupted' menstrual processes were used to explain menstrual pain in relation to FGC.

Although many participants held a primary assumption compelling them to connect young women's menstrual pain to infibulation, some medical professionals reflected upon clinical experience that suggested menstrual pain was not evidently linked to FGC but was a common complaint in all young women, with or without FGC. For example, one school nurse stated that she thought FGC could cause pain during menstruation, but was nevertheless ambivalent about whether or not there really was any difference between young women with or without FGC:

I don't have any statistics or anything, but my feeling is that young women have severe [menstrual] problems regardless. My experience is that Swedish young women I talk to also have a lot of problems during menstruation, menstrual pain that is.

Others stated that menstrual pain was a 'very common complaint' in all young women they had encountered, that they 'wouldn't be able to tell the difference' between young women with or without FGC, or that 'it is hard to distinguish from ordinary menstrual pain'. Such comments can be interpreted as partly contradicting the preunderstanding of FGC as the cause of menstrual pain in young women, as articulated by the professionals. However, these experiences seemed not to be given much explanatory power in the professionals' accounts.

\section{A Constructivist Approach to Menstrual Pain}

Given a clinical context where professionals had a preunderstanding that FGC causes menstrual pain, there was a tendency to initially connect menstrual pain reported by young women to infibulation. Accordingly, in menstruation counselling, medical professionals explained how they tried to assess whether dysmenorrhea 
was primary or secondary and, if secondary, whether pain was secondary to FGC or other conditions. In management of young women's pain, they were leaning on general medical training, particularly insight into the physiological processes associated with dysmenorrhoea, as well as knowledge gained from education specifically on FGC. However, professionals also used knowledge based on cultural expectations on adequate responses to menstrual pain and their clinical knowledge about how best to manage it.

In discussing menstrual pain in young women with presumed FGC, some clinical professionals described how they had observed that young women responded to pain in ways dismissed by the professionals as inadequate. When they discussed these experiences, they spoke more broadly about young women with a migrant background, often taking Somali young women as an example. Responses to menstrual pain, explained by the interviewees, included lying still and resting, drinking hot tea, missing school and having excessive difficulties continuing life as usual. In these descriptions, there was a tendency to talk about the young women's responses as culturally influenced. Some expressed they believed 'cultural taboos', 'stigma' or ideas about 'uncleanliness' make young women stay home from school, or that cultural expectations to 'suffer in silence' could be an answer to a young woman's unwillingness to use painkillers. Young women's responses were also discussed by some as health 'illiteracy' or 'knowledge deficiency' regarding how to interpret bodily processes or appropriate ways to respond to them. A midwife and school nurse expressed it thus:

They think that it's a sign of disease. And of course, if you lack knowledge about menstruation, and how it works and what you can do about it, then it gets difficult [for you]. Then you'll think you're ill when you have your period, and you stay out of school. And then they have mothers who ... don't have the knowledge either, who also think they're ill.

In this respect, the professionals' accounts illustrated a more constructivist approach to the young women's pain. They suggested that sociocultural factors such as the way health, bodily processes, and menstruation are understood, play a role in young women's experiences of and responses to menstrual pain. Here, excessive menstrual pain was not primarily portrayed as the result of FGC but was described as physiologically the same in all young women, albeit as something that could be handled in more or less efficient ways.

In contrast to the young women's strategies to cope with pain, medical professionals emphasised the importance of physical activity and continuing to go to school, in addition to the option of medication i.e. 'painkillers' or 'hormonal contraceptives' in treating menstrual pain. Framed in terms of their clinical knowledge and experience of efficient ways of responding to pain, common comments were along the lines: 'I tell them that, to feel better, it's important they stay physically active so that the bleeding can start', as put by a school nurse. Also, a school nurse and midwife proposed, 'You shouldn't be in such pain that you can't go to school. That's not okay'.

In these accounts, Sweden was implicitly presented as a country with high acceptance and limited stigma attached to menstruation and menstrual pain. A midwife and school nurse commented on what she considered 'the Swedish' normative 
stance on menstrual pain: "We are the opposite here, it's like "bite the bullet" and "this is how it is", or "yes, so what?, everybody has it". It has almost become the opposite [here].' 'Crying out' in pain, 'hiding' or feeling 'ashamed' during menstruation were positioned as odd. Instead, the examples illustrate that menstrual pain is not expected to be particularly troublesome or to hinder women in their daily lives, i.e. menstruating women are expected to be able to continue life as normal. Accordingly, many reasoned that pain which does hinder daily life is pathological and merits medical attention. Professionals described how they, for example, suggested defibulation (in case of infibulation) or further clinical assessment to rule out other potential causes to pain such as endometriosis, if a young woman presented with excessive pain.

A former school nurse, working in primary care at the time of the interview, however, gave a contrasting account. She commented on expressions of menstrual pain among young women with FGC:

It's possible they don't really accept it, don't know what to do and 'don't want to go to gym class' and [think] it's embarrassing [having a period]. And I think many Swedish girls also experience this, that it's difficult and they don't want to say that they have their period.

This participant focused more on similarities in expressing painful menstruation, suggesting that young women might face challenges in going on with life as normal or might be affected by cultural stigma regardless of cultural background.

In terms of menstruation counselling, professionals navigated different explanatory frameworks in their efforts to make sense of and respond to the young women's pain complaints, whether pain was thought to result from FGC and/or be an expression of an unfamiliar cultural coping strategy, or just ordinary (primary) dysmenorrhoea. Thus, on the one hand, participants described and dismissed young women's culturally different responses to menstrual pain, stressing the importance of continuing life as normal during menstruation or else seeking medical care. On the other hand, they sympathised with the young women's strategies during menstruation, acknowledging that all young women might experience stigma and challenges in continuing life as usual during menstruation.

\section{Menstrual Pain as a Working Tool in Clinical Practice}

Participants strived to acknowledge their awareness of presumed harms of FGC, and argued that FGC was an important issue to address. All had made efforts to initiate conversations about FGC in professional encounters with young women possibly affected by FGC. Efforts were made both in relation to their specific work context as well as the legal context.

For medical professionals, menstrual pain was described as a useful working tool in clinical practice, in one of two ways. First, given a clinical context where FGC was expected to lead to painful menstruation, menstrual pain seemed to function as a flexible working hypothesis alerting professionals to the possibility 
that the person before them might be infibulated. An example of this approach was expressed by a midwife at a youth clinic:

[In taking down a menstruation history] we get information about whether they have a really hard time with their period, whether they need to stay home from school, if they're helped by painkillers ... then you have that with you in the back of your mind ... and already have a plan worked out so that you can steer the conversation towards those questions [about FGC] as well.

Also in relation to social work practice, menstrual pain as a 'working hypothesis on FGC' was brought up by one social worker. She commented that after receiving education about $\mathrm{FGC}$, she was more attuned to menstrual pain as a symptom that suggested a girl or a woman may have been exposed to FGC, although she had not yet applied this information in her work. Starting from this working hypothesis, some professionals explained how they tried to assess whether the young woman might have been infibulated and, if this was the case, whether she had other health issues related to the FGC. Many described how they would first recommend or offer information about defibulation in case the young woman is infibulated. In addition, professionals explained how they follow their standard protocol, communicating the option of medication i.e. 'painkillers' or '[hormonal] contraceptives', or provide information about other ways to treat menstrual pain such as with 'heat' and 'physical activity'. Others described how they make an assessment in conjunction with providing brief information about possible harms of FGC and available options for help, including the alternative of defibulation, in the event the young woman needed it, then or forthwith. This was the case without, sometimes, knowing whether or not the young women had undergone FGC.

Second, another trend permeating the material was that many professionals in clinical practice described how they used menstrual pain as a starting point for conversations about FGC. Participants argued that the question 'fits there', or is 'a useful way in'. A typical scenario in the interviews was expressed by a school nurse in an FGD with other participants making supportive comments:

I actually start by asking about menstruation because I think it's a useful way in. 'How is it when you have your period? Are you in a lot of pain?' ... and then I continue, 'Could it be that you are circumcised?' And yes, that's often the case. Some have trouble, some don't, and those who don't [well then] it's not a problem and then they don't want help either.

The strategy for initiating conversation was influenced both by the professional and the legal context. Mindful that young women might be aware that FGC is not a universal norm and that the practice is indictable and morally condemned in Sweden, many expected FGC to be a sensitive topic to discuss. All professionals thus described efforts to raise the question in a way that would not alienate, offend or frighten the young woman. Strategies described included signalling awareness of FGC, stressing they had no intention of reporting the (previously 
performed) FGC to authorities, avoiding talking about FGC in terms of 'right and wrong', not using the word 'mutilation', and 'not being hasty' in encouraging young women to disclose their genital status, but 'letting things take their time'. While some medical professionals had implemented organisational protocols for taking FGC history during routine health check-ups [hälsosamtal] (for example to ask directly about FGC or ask all young people in health encounters if they had had genital surgery), this was not the case for most professionals in this study. Instead, those who emphasised menstrual pain as a useful way to initiate discussions about FGC often commented that they lacked other ways to raise the issue. For one school nurse, targeting FGC indirectly starting with a medical symptom, had helped her to 'have the courage to raise the question' already 'being in that area of the female body'. Yet others emphasised that an approach focused on possible symptoms (experienced or not) helps to invite a non-intimidating conversation as it signals the mere interest to offer help. A school nurse encapsulated this latter perspective:

I don't ask directly about circumcision, because I won't get a good answer. Instead, I ask if they have any trouble in their genital area ... problems with peeing, pain or painful menstruation. 'If so, I can help you' and 'you can get these kinds of problem if you have been circumcised, for example'. So I try to use this as a way into the conversation, and then I feel it's easier to reach them.

In these manners, (1) menstrual pain as a flexible working hypothesis and/or (2) menstrual pain as a tool for initiating conversations, were presented by many of the medical professionals as useful tools in clinical encounters with young women potentially exposed to FGC. Among the non-medically trained professionals, menstrual pain worked as an alert on FGC for the social worker who had been informed about such an association in FGC-specific education. For another social worker, who had no prior FGC education, no such association was made. Instead, she commented that she lacked the tools to initiate conversations. In encounters with young women that she knew had undergone FGC, she had asked generally about health problems but had not specifically targeted FGC.

As suggested by these examples, menstrual pain itself was not necessarily the primary concern, but was used as a tool to steer the conversation towards aspects which were considered important. These aspects varied. While many articulated that they wished the practice would end, describing it as 'oppressive', 'harmful' or 'unnecessary', the interviews indicated that the main goal of initiating conversations about FGC was to start communication in order to provide help and support and to pave the way for harm reduction work in the long term. Professionals reported that conversations could be about assessing whether a young woman was suffering as a result of FGC, or about providing information on health risks or potential future sexual and reproductive health problems they associated with FGC and provide information on pathways for help i.e. gynaecological assessment and defibulation then or at a later stage. A few noted that they also aimed to work preventively by providing information on legislation and potential sexual and other health risks or asked about attitudes towards FGC in order to protect future generations. This was, for example, the case with the professional working as a health educator on a strategic level, but 
also among other professionals, although prevention did not appear to be their main concern. Another, more general motive stated was to pave the way for open communication about FGC.

In sum, medical professionals used an assumed association between menstrual pain and FGC as a way of initiating conversations about FGC. The ambition, it seemed, was to build rapport by offering a friendly environment for conversations so as to be able to offer support, then or forthwith. Conversations were initiated first and foremost to pave the way for long-term harm-reduction work, and secondly, for a few, to facilitate conversations aimed at long-term prevention and protection of future generations. This strategy was employed in relation to the professional, sociopolitical and legal contexts.

\section{Discussion}

This study provided a qualitative exploration of how professionals in schools, healthcare and social services approach sexual and reproductive healthcare encounters in relation to awareness about FGC. In a context where professionals are urged to acquire knowledge about possible health effects of FGC as a way to tackle prevention and care in relation to the practices, the way professionals understand and respond to knowledge gained through campaigns and training is pertinent to explore. This study provides a novel contribution. Departing from menstruation counselling, the study shows how professionals navigate their various sources of knowledge including awareness of FGC in encounters with young women who may have been exposed to FGC. The analysis shows that there was a tendency in counselling to differentiate young migrant women's menstrual complaints from ordinary menstrual pain, with professionals understanding pain complaints either in terms of FGC or as culturally influenced. FGC awareness worked as a lens through which young women's health complaints were understood. Biomedical knowledge and culture-specific expectations and assumptions regarding menstrual pain also informed counselling. In encounters with young women potentially affected by FGC, menstrual pain was presented as a particularly valuable tool in clinical practice.

With a few exceptions, participants had taken part of FGC education and reference materials on FGC. Similar to how menstrual pain has been discussed in Swedish FGC education, policy materials and guidelines (Almroth, 2008; National Board of Health \& Welfare, 2016; Östergötland County Administrative Board, 2015), the study found that professionals with training on FGC had a preunderstanding that FGC causes menstrual pain. For these participants, menstrual pain worked as an alert and flexible working hypothesis about FGC among young women from highprevalence countries. In this respect, FGC awareness and especially the linkage made between FGC and menstrual pain, functioned as an 'interpretive resource' (Loseke, 2001) and lens through which young women's health complaints initially were understood as potentially caused by FGC.

The study shows that participants strived to acknowledge awareness of FGC in encounters with young women potentially affected by FGC, having all made efforts to raise the issue. Efforts were made both in relation to the professional and legal 
context. In a context where FGC is unlawful and stigmatised, participants expected FGC to be a sensitive topic to discuss, and navigated ways to use the knowledge in a sensitive, non-judgemental and non-intimidating way. The study found that, against this background, professionals used the presumed association between menstrual pain and FGC as an indirect intake to conversations about FGC. Previous literature has linked professionals' unease to broach the topic of FGC to having more to do with the professionals' assumptions about the practices (Leonard, 2020) or 'outsiders' own moral and emotional difficulties' with FGC, than with actual experiences in professional practice (Johansen, 2006, 523). In this study, professionals formulated their concerns in terms of finding their way to initiate a discussion in ways that would not alienate, offend or frighten the young woman. Professionals are welladvised in their caution not to use stigmatising language and attitudes, as this might contribute to distrust in service providers (Khaja et al., 2010) and influence willingness to seek care (Michlig et al., 2021), as well as contributing to women feeling 'abnormal' (Villani, 2020), 'traumatised' or 'stigmatised' (Karlsen et al., 2019). Starting conversations about health problems (experienced or not) that were believed to resonate with the real-life experience of living with FGC, was seen as a tool for building rapport and establishing a neutral, less intimidating environment. The strategy can be seen as an effort to give 'permission to talk' as termed in sexual health counselling (Annon, 1976) and pave way for open communication about FGC. For professionals who had implemented guidelines for raising the issue directly, the approach of targeting FGC indirectly was not used but should be viewed in relation to the professional context. The strategy was also related to the professionals' individual agenda. While participants to some extent intended to work preventively by providing information about legislation or potential harms of FGC, the main goal, as it appeared, was to build trust and pave the way for better care provision in the long term. Starting conversations about medical symptoms was seen as a way to signal an intention to help. With regard to the impact of policy and interventions, the findings contrast with what was reported in a recent study of British-Somali women that suggests healthcare professionals used FGC awareness to extract information for safeguarding reasons 'over and above [the women's] health needs' (Karlsen et al., 2019, 7). In the UK, healthcare professionals have an absolute duty to report any FGC identified in underaged young women. This is not the case in Sweden, even though the national organ responsible for FGC education in Sweden has encouraged reporting FGC in all minors to authorities. ${ }^{7}$ From a policy and intervention perspective, the results call for further consideration of the role of the professional, socio-political and legal context in which professional practices take place.

\section{Menstrual Pain as Physiology and Culture}

While the present study found menstrual pain was used as a tool in clinical practice, the study shows that professionals struggled to make sense of menstrual pain

\footnotetext{
7 Official website of the organ responsible for the national work on FGC in Sweden, hedersfortryck.se/ hedersrelaterat-vald-och-fortryck/konsstympning.
} 
in young women that may have been exposed to FGC. On the one hand, a main idea was that a narrow vaginal opening (infibulation) causes an obstruction of menstrual flow, subsequently disturbing menstruation processes and causing pain in various ways. This finding aligns with how menstrual pain in relation to FGC has been framed in Swedish information and training on FGC (e.g. Almroth, 2008; National Board of Health \& Welfare, 2016; Östergötland County Administrative Board, 2015). The finding suggests that professionals had adopted messages from FGC training even though research on menstrual pain and FGC has not been able to prove the correlation (e.g. Lurie et al. 2020; Obermeyer, 2005). Medical professionals also supported their understanding on knowledge based in medical training on physiological processes associated with primary and secondary dysmenorrhoea. On the other hand, concurrently, professionals employed a constructivist approach to pain, and tended to refer to young migrant women's responses to menstrual pain as an expression of culture (e.g. shame, stigma, or taboo) or health illiteracy, in a sense positioning them as based in a traditional, as opposed to a modern, biomedical knowledge system. The way of understanding young women's menstrual pain in terms of either FGC and disturbed physiological processes, or as a culturally influenced expression, suggests a differentiation of young migrant women's expressions of menstrual pain from ordinary menstrual pain. The framing of young migrant women's responses to pain (i.e. staying home from school, having excessive difficulties continuing life as usual) as excessive and deviant, as opposed to normal, suggests a tendency to culturalise the young women's expressions of pain. At the same time, the differentiation of young migrant women's menstrual pain from 'normal' menstrual pain, was brought into question when professionals drew on their clinical observations and alluded to menstrual pain as a common complaint in all young women, also conceding that all young women may struggle with stigma or shame in relation to menstrual pain. These experiences, however, did not appear to be given explanatory power in the professionals' efforts to make sense of the young women's menstrual pain. In the context of existing studies on adolescents' coping strategies for menstrual pain demonstrating that staying home from school and resting are strategies common across many ethnic groups (De Sanctis et al., 2016), the professionals' approach, suggesting that menstruating women can and should take part in activities as usual, can be seen as participants giving voice to a Swedish cultural 'script' on menstrual pain. According to Simon and Gagnon (1986), cultural scripts inform individuals about cultural codes concerning how to enact and interpret their experiences and lives. The sentiments in the interviews reveal prevailing assumptions that menstrual pain is not expected to disrupt women's daily lives, or else the pain is pathologised. Recent campaigning from menstrual activists and governmental public-health initiatives further suggests that this is an established idea, and script, in Sweden. For example, in the campaign 'severe menstrual pain is not OK!' by the National Board of Health and Welfare, the main message is that menstrual pain that leads to absenteeism requires medical assessment and treatment (National Board of Health \& Welfare, 2019). In contrast, menstrual activists in Sweden are making efforts to change 
current framing of menstrual pain, claiming it is not to be taken lightly and that it justifies sick-leave or adapting workload. ${ }^{8}$

Counselling on menstrual pain was thus based not only on professionals' awareness of FGC and their medical training on dysmenorrhoea, but also on their culture-specific expectations about adequate ways to express and respond to menstrual pain. In light of scripts (Simon \& Gagnon, 1986), it can be interpreted as professionals navigating different cultural scripts and norm systems in menstruation counselling with young women from a cultural background different from their own. In doing this work, they leaned on information picked up in FGC training, their medical training and also on cultural norms specific to menstrual pain. On a normative level, the inclination to repudiate young migrant women's coping strategies, or scripts, as 'inadequate' or health 'illiteral', suggests professionals' own knowledge system and frames of understanding were given a superior position. Yet, on an individual level, in menstruation counselling, participants sympathised with young women's pain, and employed a variety of explanatory frameworks to make sense of and respond to young women's lived experiences of menstrual pain.

\section{Implications for Practice}

Awareness of FGC and the linkage made between FGC and possible health effects, especially menstrual pain, encouraged professionals to be attentive to the possibility of FGC among young women from high-prevalence countries presenting with health complaints. A central question then is, to what extent is the FGC awareness of help in efforts to establish better communication and rapport in service encounters?

In a wider context, the linkage made between menstrual pain and FGC can be viewed in light of a long-term engagement in FGC-intervention that has been taken on by different actors worldwide (e.g. WHO et al., 2008). In this apparatus, a standardised way to frame FGC has appeared, a 'formula story' (Loseke, 2001), or a grand narrative that includes a familiar narrative about the four types of FGC operations, what they entail, why the practice exists and its possible consequences for health and sexuality (Leonard, 2000, 2020). Such descriptions have been referred to as 'the standard tale' of FGC as they are often represented in a repetitive manner (Leonard, 2000). Among critical scholars, however, there is a growing consensus that many popular beliefs about FGC are misguided and rest on weak empirical assumptions (Earp \& Johnsdotter, 2021; Obermeyer, 2005; PPAN 2012; Shell-Duncan, 2008). Many have also noted that there is a tendency to relate to the most extensive type (infibulation) in descriptions about FGC, which might contribute to misconceptions about FGC in contexts where lesser forms are prevailing (e.g. Johansen, 2006). This is relevant not least in the Swedish context where FGC type III used to be a common type of FGC among immigrants, but now is a practice in decline (Powell et al., 2020), which is also suggested by the observations made by professionals in this

\footnotetext{
8 www.mensen.se, project 'menstruation-certified workplaces', [menscertifiering av arbetsplatser] launched in 2019, information extracted 1 July 2020.
} 
study. Moreover, recent studies suggest that it is not necessarily the extent of the procedure that determines how harmful it is perceived by the individual to be (if experiencing harm at all), but the experience of the procedure itself (Michlig et al., 2021; see also Sharif Mohamed et al., 2020).

What problems may arise if such a 'standard tale', based on sometimes uncertain empirical assumptions, is used in professional practice? Insofar as the knowledge contributes to establishing trust or better care and communication about FGC, it appears to be a valuable tool. The results show that awareness about possible health risks was used constructively as a way to build rapport and establishing a dialogue about FGC, and for many the strategy seemed to work fine. In addition, an unintended consequence seems to be that complaints about menstrual pain were acknowledged to a great(er) extent. There are, however, possible pitfalls with this approach, some of which will be discussed below.

First, with a lack of compelling alternatives, the strategy among some professionals to target FGC indirectly through menstrual pain, suggests that young women are given unequal opportunity to talk about FGC-related issues, when such conversations are made dependent on the event of particular health complaints. This is not necessarily a problem. Some might argue that if the primary focus is on care and support, raising the issue would only be relevant if the young woman has concerns or health issues that could be related to the FGC. On the other hand, from the perspective of safeguarding, or harm reduction in the long term, an opportunity to discuss FGC might get lost with such an approach.

Secondly, as has been argued elsewhere (Earp, 2020; Palm et al., 2019; Sharif Mohamed et al., 2020), while it is essential to acknowledge potential risks of FGC, a reductionist focus on the (cut) genitals, or jumping to the conclusion that health complaints must be related to the FGC, might lead to underdiagnosis or undertreatment of other conditions that may be related to the woman's complaints. Such a reductionist gaze, directed too narrowly on the stigmatised or non-normative identity or experience, is not limited to healthcare encounters with women with FGC (e.g. Bergström et al., 2013; Khaja et al., 2010) but has been identified in several sexuality related fields. For example, in a Swedish context such an approach has been reported in relation to LBGT groups (Björkman \& Malterud, 2009), persons with experience of selling sex (Holmström, 2015; Larsdotter et al., 2011), and people with polyamorous lifestyles (Carlström \& Andersson, 2019). Ultimately it may lead to suboptimal outcomes of the health encounter. A contrasting approach in the clinical encounter, as suggested by some interviewees in this study, is to start with an unbiased clinical assessment before giving recommendations, bearing in mind that FGC can, but does not necessarily have to, play a part in the person's illness (Johnsdotter \& Essén, 2020). This is pertinent not the least in the context of uncertain evidence as regards menstrual pain and FGC (Lurie et al., 2020; Obermeyer, 2005). In such a context, the intention to proceed from young women's real-life experiences of FGC, seems misplaced. Counter to the intention, an overemphasis on FGC as the cause of complaints could possibly diminish trust and dialogue and willingness to seek help, if the young woman feels singled out or perceives counselling as based on misconceptions about FGC or her situation (Bergström et al., 2013; Karlsen et al., 2019; Khaja et al., 2010). Thus, for all that is good with increased awareness of FGC, to counter the event that 
professional practice is based on stereotypes or misguided assumptions about FGC and the people affected by it, the results in this study stress the importance of wellinformed interventions and, thus, rigorous research and quality-checking in the first place (see also Essén \& Mosselmans, 2021).

Third, a downside of introducing FGC as a probable cause for discomfort is the risk of iatrogenic pathology (Michlig et al., 2021) in that it might prompt young women to interpret their bodies in terms of deficiencies related to FGC (and, as such, a chronic condition), when this does not correspond to their own experiences, or when menstrual pain might just be ordinary dysmenorrhoea (thus temporary). Ultimately, through expectancy effect, this could lead to maladaptive responses or internalisation of genital pain (see Earp, 2020 for a theoretical discussion). Reading one's body in terms of 'mutilation', deficiency or pain, is not common in local contexts where FGC is the norm. Although people often are aware of the risk of serious health consequences (Shell-Duncan, 2008), genital modifications are typically associated with beautification, morality, or sometimes seen as a preparation for the ordeals of life (Jirovsky, 2014; Malmström, 2013). In western migration settings, however, studies have shown that internalising new ideas about FGC has come to shape women's perceptions, not only about the practices, but also in terms of body image, sexual self-image and perceptions of genital pain (Johansen, 2007; Lien and Schultz 2013; Villani, 2020). Professionals in this study were well aware and cognisant of these risks and used strategies to destigmatise young women's experiences of FGC. Again, though, if not based in well-informed policies and interventions, there is a risk that professionals' strategies fall short, however carefully chosen and well-intended they are. Some might argue, from this perspective, that current 'awareness' might serve as a hinderance for optimal care and prospects for establishing trust.

\section{Methodological Considerations and Implications for Future Research}

Participants included professionals who had experience of encounters related to FGC, and the vast majority had taken part in FGC education. Participants' accounts could therefore result from a vested interest in these issues and could perhaps reflect perspectives grounded in professional experience to a greater extent than would be the case among individuals who did not participate.

This study, which includes personnel with varied professional backgrounds, underscores the importance of considering the particular contexts of professionals in understanding how they understand and make use of acquired knowledge about FGC. These findings are important, not least in a context such as Sweden, where non-medically trained staff are urged to be attentive to medical conditions such as menstrual pain in order to identify girls and young women with FGC, either as a safeguarding or preventive measure, or in order to provide care and support (National Board of Health \& Welfare, 2016; Östergötland County Administrative Board, 2015).

Future studies should explore the role of professionals' experiences of clinical encounters, in relation to how FGC policy and interventions are interpreted and 
implemented in practice. A hypothesis based on the findings of this article is that where professionals have less experience of clinical encounters, policy and interventions will play a greater role in clinical practice. Given the inconsistent evidence on sexual and reproductive health effects of FGC (Andro et al., 2014; Obermeyer, 2005), the study also raises questions about what knowledge professionals should acquire, and what work professionals $d o$ with the knowledge gained. These questions merit further critical scrutiny. Another area for future research is to explore, in more depth, how sociocultural ways of framing the circumcised body, pain, and claimed consequences for sexual health affect how girls and women with FGC reflect on their bodies post-migration to the West.

Acknowledgements This work was supported by the Swedish Research Council for Health, Working Life and Welfare (FORTE) under Grant 2015-00771; the Swedish Research Council (Vetenskapsrådet) under Grant 2015-03546, and Malmö University.

Author Contributions B.E. and S.J. obtained funding and administered the project. C.P., B.E. and S.J. designed the research and C.P. collected and analysed the qualitative data. C.P. wrote the manuscript which was supervised, revised, and edited by E.E. and C.H. C.P., E.E. and C.H. contributed to the conceptualisation of the analysis. All authors read and approved the final manuscript.

Funding Open access funding provided by Malmö University.

Data Availability All relevant data are included in the article.

\section{Declarations}

Conflict of interest No potential conflict of interest was reported by the authors.

Informed Consent Informed consent was obtained from all individual participants included in the study.

Ethical Standards The research was conducted in accordance with the ethical standards of the National Research Committee, and with the 1964 Helsinki Declaration and its later amendments or comparable ethical standards.

Open Access This article is licensed under a Creative Commons Attribution 4.0 International License, which permits use, sharing, adaptation, distribution and reproduction in any medium or format, as long as you give appropriate credit to the original author(s) and the source, provide a link to the Creative Commons licence, and indicate if changes were made. The images or other third party material in this article are included in the article's Creative Commons licence, unless indicated otherwise in a credit line to the material. If material is not included in the article's Creative Commons licence and your intended use is not permitted by statutory regulation or exceeds the permitted use, you will need to obtain permission directly from the copyright holder. To view a copy of this licence, visit http://creativecommons.org/licen ses/by/4.0/.

\section{References}

Abdi, R. (2012). Carving culture. Creating identity through female genital cutting. Durham Anthropology Journal, 18(1), 115-153.

Abdulcadir, J., Botsikas, D., Bolmont, M., et al. (2016). Sexual anatomy and function in women with and without genital mutilation: A cross-sectional study. Journal of Sexual Medicine, 13(2), 226-237. 
Abdulcadir, J., Margairaz, C., Boulvain, M., et al. (2011). Care of women with female genital mutilation/ cutting. Swiss Medical Weekly, 140, w13137.

ACOG. (2021). Dysmenorrhea: Painful periods. The American College of Obstetricians and Gynecologists (ACOG).

Ahmadu, F. (2000). Rites and wrongs: An insider/outsider reflects on power and excision. In B. ShellDuncan \& Y. Hernlund (Eds.), Female “circumcision” in Africa: Culture, controversy, and change (pp. 283-315). Lynne Rienner Publishers.

Almroth, L. (2008). Komplikationer vid könsstympning [Complications of female genital mutilation]. In V. Berggren \& M. Franck (Eds.), Kvinnlig könsstympning. [Female genital mutilation] (pp. 87-103). Malmö: Studentlitteratur.

Alsibiani, S. A., \& Rouzi, A. A. (2010). Sexual function in women with female genital mutilation. Fertility and Sterility, 93, 722-724.

Andro, A., Cambois, E., \& Lesclingand, M. (2014). Long-term consequences of female genital mutilation in a European context: Self perceived health of FGM women compared to non-FGM women. Social Science \& Medicine, 106, 177-184.

Annon, J. (1976). The PLISSIT model: A proposed conceptual scheme for the behavioral treatment of sexual problems. Journal of Sex Education and Therapy, 2(1), 1-15.

Arafa, A. E., Elbahrawe, R. S., Shawky, S. M., et al. (2018). Epidemiological and gynecological correlates with female genital mutilation among Beni-Suef University students; cross sectional study. Middle East Fertility Society Journal, 23, 184-188.

Berg, R. C., \& Denison, E. (2012). Does female genital mutilation/cutting (FGM/C) affect women's sexual functioning? A systematic review of the sexual consequences of FGM/C. Sex Res Soc Policy, 9(1), 41-56.

Bergström, A. R., Nur, F., \& Davis, D. L. (2013). Consider the personhood of women who experienced genital cutting. Letter to the Editor Mayo Clinic Proceedings, 88(10), 1180.

Björkman, M., \& Malterud, K. (2009). Lesbian women's experiences with health care: A qualitative study. Scandinavian Journal of Primary Health Care, 27, 238-243.

Bobel, C., Winkler, I. T., Fahs, B., et al. (2020). The palgrave handbook of critical menstruation studies. Springer.

Braun, V., \& Clarke, V. (2006). Using thematic analysis in psychology. Qualitative Research in Psychology, 3(2), 77-101.

Brussels Collaboration on Bodily Integrity. (2019). Medically unnecessary genital cutting and the rights of the child: Moving toward consensus. American Journal of Bioethics, 19(10), 17-28.

Carlström, C., \& Andersson, C. (2019). Living outside protocol: Orientations, bodies and queer temporalities. Sexuality \& Culture, 23(4), 1315-1331.

Carven, S., Kavanagh, A., \& Khavari, R. (2016). Female genital mutilation management in the ambulatory clinic setting: A case study and review of the literature. Journal of Surgical Case Reports, 6, $1-3$.

Catania, L., Abdulcadir, O., Puppo, V., et al. (2007). Pleasure and orgasm in women with female genital mutilation/cutting (FGM/C). The Journal of Sexual Medicine, 4(6), 1666-1678.

Chalmers, B., \& Hashi, K. O. (2000). Somali women's birth experiences in Canada after earlier female genital mutilation. Birth, 27(4), 227-234.

De Sanctis, V., Soliman, A. T., Elsedfy, H., et al. (2016). Dysmenorrhea in adolescents and young adults: A review in different countries. Acta Bio-Medica, 87(1), 000-000.

De Sanctis, V., Soliman, A., Bernasconi, S., et al. (2015). Primary dysmenorrhea in adolescents: Prevalence, impact and recent knowledge. Pediatric Endocrinology Reviews, 13(2), 465-473.

Dopico, M. (2007). Infibulation and the orgasm puzzle: Sexual experiences of infibulated Eritrean women in rural Eritrea and Melbourne Australia. In Y. Hernlund \& B. Shell-Duncan (Eds.), Transcultural bodies: Female genital cutting in global context (pp. 224-247). Rutgers University Press.

Earp, B. (2020). Protecting children from medically unnecessary genital cutting without stigmatizing women's bodies: implications for sexual pleasure and pain [Commentary]. Archives of Sexual Behaviour.

Earp, B., \& Johnsdotter, S. (2021). Current critiques of the WHO policy on female genital mutilation. IJIR, 33, 196-209.

El-Defrawi, M. H., Lotfy, G., Dandash, K. F., et al. (2001). Female genital mutilation and its psychosexual impact. Journal of Sex and Marital Therapy, 27, 465-473.

Elnashar, A., \& Abdelhady, R. (2007). The impact of female genital cutting on health of newly married women. International Journal of Gynecology and Obstetrics, 97, 238-244. 
Essén, B., \& Johnsdotter, S. (2017). Is research data used in education for health professionals on management of female genital cutting? Results from Sweden. Reproductive Health, 14(suppl 2), 115.

Essén, B., \& Mosselmans, L. (2021). How to ensure policies and interventions rely on strong supporting facts to improve women's health: The case of female genital cutting, using Rosling's Factfulness approach. Acta Obstetricia Et Gynecologica Scandinavica, 100(4), 579-586.

Fajrin, I., Alam, G., \& Nilwati Usman, A. (2020). Prostaglandin level of primary dysmenorrhea pain sufferers. Enfermería Clínica, 30(S2), 5-9.

Harel, Z. (2012). Dysmenorrhea in adolescents and young adults: An update on pharmacological treatments and management strategies. Expert Opinion on Pharmacotherapy, 13(15), 2157-2170.

Holmström, C. (2015). Förbud mot köp av sexuell tjänst i Sverige - en kunskapsöversikt om avsedda konsekvenser. [Prohibition against buying sexual services in Sweden-a review of intended consequences]. The Swedish Association for Sexuality Education (RFSU).

Iacovides, S., Avidon, I., \& Baker, F. C. (2015). What we know about primary dysmenorrhea today: A critical review. Human Reproduction Update, 21(6), 762-778.

Iavazzo, C., Sardi, T. A., \& Gkegkes, I. D. (2013). Female genital mutilation and infections: A systematic review of the clinical evidence. Archives of Gynecology and Obstetrics, 287, 1137-1149.

Jirovsky, M. E. (2014). Contemporary meanings of female circumcision/female genital mutilation (FC/ FGM) in Bobo-Dioulasso, Burkina-Faso - Local aspects of a global assemblage [dissertation]. University of Vienna.

Johansen, R. E. B. (2007). Experiencing sex in exile: Can genitals change their gender? In Y. Hernlund \& B. Shell-Duncan (Eds.), Transcultural bodies: Female genital cutting in global context (pp. 224247). Rutgers University Press.

Johansen, R. E. B. (2006). Care for infibulated women giving birth in Norway: An anthropological analysis of health workers' management of a medically and culturally unfamiliar issue. Medical Anthropology Quaterly, 20(4), 516-544.

Johnsdotter, S. (2018). The impact of migration on attitudes to female genital cutting and experiences of sexual dysfunction among migrant women with FGC. Current Sexual Health Report, 10, 18-24.

Johnsdotter, S. (2019). Meaning well while doing harm: Compulsory genital examinations in Swedish African girls. Sexual and Reproductive Health Matters, 27(2), 87-99.

Johnsdotter, S., \& Essén, B. (2020). Deinfibulation contextualized: delicacies of shared decision-making in the clinic [Commentary]. Archives of Sexual Behavior.

Kaplan, A., Forbes, M., Bonhoure, I., et al. (2013). Female genital mutilation/cutting in The Gambia: Long-term health consequences and complications. International Journal of Women's Health, 5, 323-331.

Karlsen, S., Carver, N., Mogilnicka, M., et al. (2019). When safeguarding becomes stigmatising: A report on the impact of FGM-safeguarding procedures on people with a Somali heritage living in Bristol. Bristol University.

Khaja, K., Lay, K., \& Boys, S. (2010). Female circumcision: Toward an inclusive practice of care. Health Care for Women International, 31, 686-699.

Latthe, P., Latthe, M., Say, L., et al. (2006). WHO systematic review of prevalence of chronic pelvic pain: a neglected reproductive health morbidity. BMC Public Health, 6, 177.

Larsdotter, S., Jonsson, J., \& Gäredal, M. (2011). Osynliga synliga aktörer, Hbt-personer med erfarenheter av att sälja och/eller köpa sexuella tjänster. [Invisible visible actors, LGBT people with experiences of selling and/or buying sexual services]. The Swedish Federation for Lesbian, Gay, Bisexual, Transgender, Queer and Intersex Rights (RFSL). Stockholm: RFSL.

Lien, I-L., \& Schultz, J-H. (2013). Internalizing knowledge and changing attitudes to female genital cutting/mutilation. Obstetrics and Gynecology International, 1-10.

Leonard, L. (2000). "We did it for pleasure only": Hearing alternative tales of female circumcision. Qualitative Inquiry., 6(2), 212-228.

Leonard, L. (2020). Cutting cultures: Affective attachments and clinical conundrums [Commentary]. Archives of Sexual Behavior.

Loseke, D. (2001). Lived realities and formula stories of 'battered women.' In J. A. Holstein \& J. Gubrium (Eds.), Institutional selves: Troubled identities in a postmodern world (pp. 107-126). Oxford University Press.

Lurie, J. M., Weidman, A., Huynh, S., et al. (2020). Painful gynecologic and obstetric complications of female genital mutilation/cutting: a systematic review and meta- analysis. PLoS Medicine, 17(3), e1003088. 
Mahmoud, M. I. H. (2016). Effect of female genital mutilation on female sexual function, Alexandria Egypt. Alexandria Journal of Medicine, 52, 55-59.

Malmström, M. (2013). The production of sexual mutilation among Muslim women in Cairo. Global Discourse, 3(2), 306-321.

Michlig, G., Warren, N., Berthe, M., et al. (2021). Female genital mutilation/cutting among Somali women in the U.S. State of Arizona: Evidence of treatment access, health service use and care experiences. International Journal of Environmental Research and Public Health, 18, 3733.

Ministry of Health and Social Affairs. (2018). Handlingsplan mot könsstympning av flickor och kvinnor [Action plan against genital mutilation of girls and women]. Socialdepartementet. Regeringskansliet.

Momoh, C. (2004). Female genital mutilation. Current Opinion in Obstetrics and Gynecology, 16, $477-480$.

Momoh, C., Ladhani, S., Lochrie, D. P., et al. (2001). Female genital mutilation: analysis of the first twelve months of a Southeast London specialist clinic. British Journal of Obstetrics and Gynaecology, 108, 186-191.

Morison, L., Scherf, C., Ekpo, G., et al. (2001). The long-term reproductive health consequences of female genital cutting in rural Gambia: A community-based survey. Tropical Medicine and International Health, 6(8), 643-653.

National Board of Health and Welfare. (2016). Kvinnlig könsstympning - ett stöd för hälso- och sjukvårdens arbete [Female genital mutilation - guidelines for healthcare]. Socialstyrelsen.

National Board of Health and Welfare. (2019). Mycket mensvärk är inte OK. Information till dig som arbetar i vården. [Severe menstruation pain is not OK. Information for healthcare personnel]. Socialstyrelsen.

National Board of Health and Welfare. (2020). Inventering av vård för kvinnor och flickor som har varit utsatta för könsstympning. Delrapport 1. [Inventory of care for women and girls who have been subjected to genital mutilation.]. Socialstyrelsen.

Nour, N. M. (2004). Female genital cutting: Clinical and cultural guidelines. Obstetrical \& Gynecological Survey, 59(4), 272-279.

Nour, N. M. (2008). Female genital cutting: A persisting practice. Reviews in Obstetrics \& Gynecology, 1(3), 135-139.

Obermeyer, C. M. (2005). The consequences of female circumcision for health and sexuality: an update on the evidence. Culture Health \& Sexuality, 7(5), 443-461.

Östergötland County Administrative Board. (2015). Våga se. En vägledning för stöd, vård och skydd av flickor och kvinnor som är eller riskerar att bli könsstympade [Dare to see. Guidelines to support, care for and protect girls and women who are affected or risk being genitally mutilated]. Report no 2015:16. Länsstyrelsen Östergötland.

Östergötland County Administrative Board. (2016). Uppdrag att sprida Våga göra skillnad - Slutredovisning. [Commission to spread Dare to make a difference - Final report]. Report no 2016:05. Länsstyrelsen Östergötland.

Palm, C., Essén, B., \& Johnsdotter, S. (2019). Sexual health counselling targeting girls and young women with female genital cutting in Sweden: Mind-body dualism affecting social and health care professionals' perspectives. Sexual and Reproductive Health Matters, 27(1), 1-11.

Powell, R. A., Yussuf, M., Shell-Duncan, B., et al. (2020). Exploring the nature and extent of normative change in FGM/C in Somalialand. Evidence to end FGM/C: Research to help girls and women thrive. Population Council.

PPAN, The Public Policy Advisory Network on Female Genital Surgeries in Africa. (2012). Seven things to know about female genital surgeries in Africa. Hastings Center Report, 6, 19-27.

Reig-Alcaraz, M., Siles-González, J., \& Solano-Ruiz, C. (2016). A mixed-method synthesis of knowledge, experiences and attitudes of health professionals to female genital mutilation. Journal of Advanced Nursing, 72, 245-260.

Sharif Mohamed, F., Wild, V., Earp, , et al. (2020). Clitoral reconstruction after female genital mutilation/cutting: A review of surgical techniques and ethical debate. Journal of Sexual Medicine, 17(3), 531-542.

Shell-Duncan, B. (2008). From health to human rights: Female genital cutting and the politics of intervention. American Anthropology, 110(2), 225-236.

Shiferaw, M. T., Wubshet, M., \& Tegabu, D. (2014). Menstrual problems and associated factors among students of Bahir Dar University, Amhara National Regional State, Ethiopia: A cross-sectional survey. Pan African Medical Journal, 17, 24. 
Simon, W., \& Gagnon, J. H. (1986). Sexual scripts: Permanence and change. Archives of Sexual Behavior, 15(2), 97-120.

Söderman, L., Edlund, M., \& Marions, L. (2019). Prevalence and impact of dysmenorrhea in Swedish adolescents. Acta Obstetricia Et Gynecologica Scandinavica, 98, 215-221.

Thierfelder, C., Tanner, M., \& Bodian, C. K. (2005). Female genital mutilation in the context of migration: Experience of African women with the Swiss health care system. European Journal of Public Health, 15(1), 86-90.

UNICEF. (2013). Female genital mutilation/cutting. A statistical overview and exploration of the dynamics of change. UNICEF.

Villani, M. (2020). Reconstructing sexuality after excision: The medical tools. Medical Anthropology, 39(3), 269-281.

Villani, M., Griffin, J. L., \& Bodenmann, P. (2016). In their own words: the health and sexuality of immigrant women with infibulation living in Switzerland. Soc Sci, 5, 71.

WHO. (2016). WHO guidelines on the management of health complications from female genital mutilation. World Health Organization.

WHO. (2018). Care of girls and women living with female genital mutilation. A clinical handbook. Geneva: World Health Organization.

WHO. (2020). Female genital mutilation. Fact sheet. 3 February 2020.

WHO. (2021). Health risks of female genital mutilation ( $F G M$ ). World Health Organization.

WHO, Unaids, UNPD, , et al. (2008). Eliminating female genital mutilation: An interagency statement. World Health Organization.

Publisher's Note Springer Nature remains neutral with regard to jurisdictional claims in published maps and institutional affiliations.

\section{Authors and Affiliations}

\section{Camilla Palm ${ }^{1,2}$ (1) $\cdot$ Sara Johnsdotter ${ }^{1} \cdot$ Eva Elmerstig $^{1} \cdot$ Charlotta Holmström $^{1}$. Birgitta Essén ${ }^{2}$}

1 Department of Social Work, Faculty of Health and Society, Centre for Sexology and Sexuality Studies, Malmö University, 20506 Malmö, Sweden

2 Department of Women's and Children's Health (IMCH), Uppsala University, 75185 Uppsala, Sweden 